\title{
Decrease uncertainty of measuring small differential signal against large common-mode signal
}

\author{
Pavel Baranov ${ }^{1, *}$, Valeriy Borikov ${ }^{1}$, Edvard Tsimbalist ${ }^{1}$, and Bien Bui Duc ${ }^{1}$ \\ ${ }^{1}$ Tomsk Polytechnic University, 634050 Tomsk, Russia
}

\begin{abstract}
Comparators capable of comparing two alternating signals of the similar frequency within a wide dynamic range of frequencies and voltages are lock-in amplifiers with a differential input. The paper presents the methods and means are suggested for reducing the measurements uncertainty conditioned by the finite value of the common-mode rejection ratio in lock-in amplifier with a differential input.
\end{abstract}

\section{Introduction}

A comparison of the alternating voltage values is the classical problem of instrumentation [1-2]. A method of measurements by comparison against an actual measure is commonly used providing a comparison of the value to be measured $u_{x}(t)$ and the value of calibration signal $u_{0}(t)$ reproduced by measure. The practical implementation of this method including bridge and differentiation circuits requires the availability of high-sensitivity comparators possessing a resolution that, in large part, defines the uncertainty of measurements [3-8].

Comparators capable of comparing two alternating signals of the similar frequency within a wide dynamic range of frequencies and voltages are lock-in amplifiers with a differential input [9-11]. These devices are intended for the identification of a small differential signal of voltage to be compared (up to nanovolt units) against a large commonmode signal at a signal/noise ratio of $-100 \mathrm{~dB}$ and high impedance (up to tens and hundreds of megaohms) at measurement inputs [12-13].

In this work, the methods and means are suggested for reducing the measurements uncertainty conditioned by the finite value of the common-mode rejection ratio in lock-in amplifier with a differential input.

\section{Tracking power supply for differential signal extraction}

The output voltage of the simplest lock-in amplifiers with a differential input can be obtained from:

${ }^{*}$ Corresponding author: $\underline{b p f @ t p u . r u}$ 


$$
\Delta U \approx \frac{1}{E}\left(\lim _{T \rightarrow \infty} \frac{1}{T} \int_{0}^{T}\left[u_{x}(t)-u_{0}(t)+\frac{u_{x}(t)+u_{0}(t)}{2 K_{\mathrm{RR}}(f)}\right] u_{\mathrm{ref}}(t) d t\right)
$$

where $E$ is the denominator of synchronous detector, V;

$u_{0}(t), u_{x}(t)$ are input voltages to be compared, $\mathrm{V}$;

$u_{\text {ref }}(t)$ is the reference voltage, $\mathrm{V}$;

$K_{\mathrm{RR}}$ is the common-mode rejection ratio.

From (1), measurement of the voltage difference $\Delta U$ of $u_{0}(t)$ and $\left.u_{x}(t)\right)$ is accompanied by the uncertainty that depends upon the phase shift $\varphi$ between input signals and the finite value of the common-mode rejection ratio $K_{\mathrm{RR}}$ of the comparator circuit (subtractor). Also, it depends on incoherence of reference $u_{\text {ref }}(t)$ and detected voltages at the inputs of the synchronous detector that is characterized by the phase shift $\varphi_{\text {ref. }}$ The requirements for the common-mode rejection ratio must be improved, in particular, when checking and calibrating the scaling measurement transducers at $(1-10 \mathrm{nV})$ maximum resolution of the lock-in amplifier within the medium frequency range at the higher level of $10 \sqrt{2} \mathrm{~V}$ of compared voltage dynamic range.

The increase of the common-mode rejection can be implemented by the addition of the voltage follower to the circuit as shown in Figure 1 that provides the tracking power supply of the instrumentation amplifier.

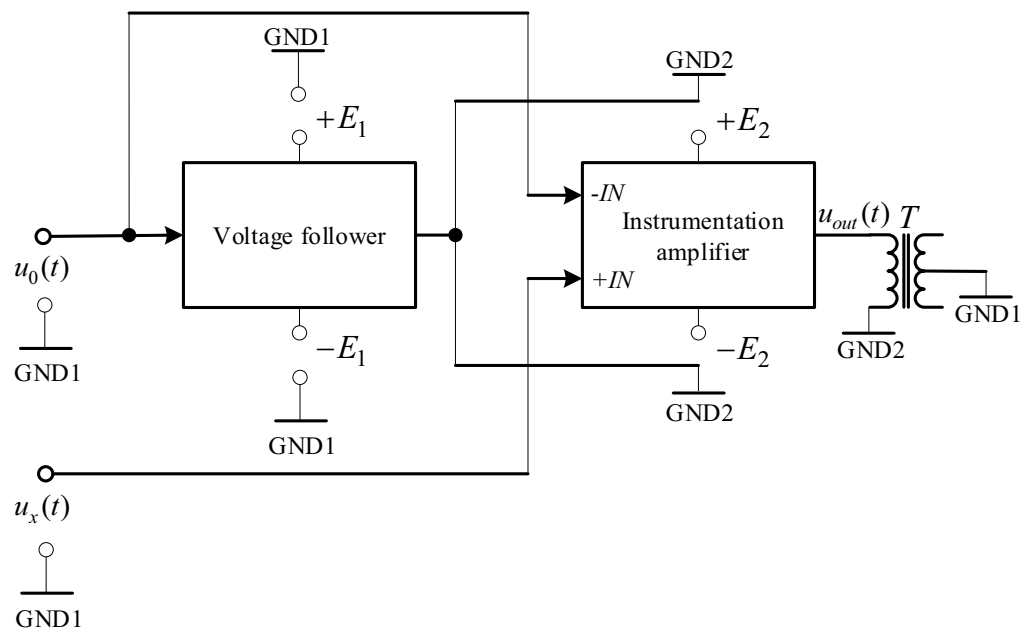

Fig. 1. Voltage follower-added circuit for formation of the tracking power supply.

For this circuit, the efficient common-mode rejection ratio will be increased and comes to

$$
K_{\mathrm{RR} . \mathrm{ef}}(f)=\frac{K_{\mathrm{RR}}(f)}{1-K_{\mathrm{f}}(f)},
$$

where $K_{\mathrm{f}}$ is the transmission factor of the voltage follower.

The common-mode rejection down to $160-180 \mathrm{~dB}$ can be achieved within the frequency range of practically inertialess circuits, at easily reachable values of $0,999-0,9999$ of the follower transmission as shown in Figure 2. These values provide the stability of the voltage follower transmission factor at different signal sources.

These dependencies prove a certain drawback of this technique used in the wide frequency range: the transmission factor module of the voltage follower and 
instrumentation amplifier decreases with the increase of frequency resulting in the common-mode rejection ratio of the whole circuit. Therefore, the high rejection of the common-mode signal is observed only in the narrow band of frequencies.

It should be noted that tracking power supply circuit with the use of voltage follower provides also a jump of the input impedance of measuring channels in the wide frequency range, especially $u_{x}$ channel of the instrumentation amplifier.

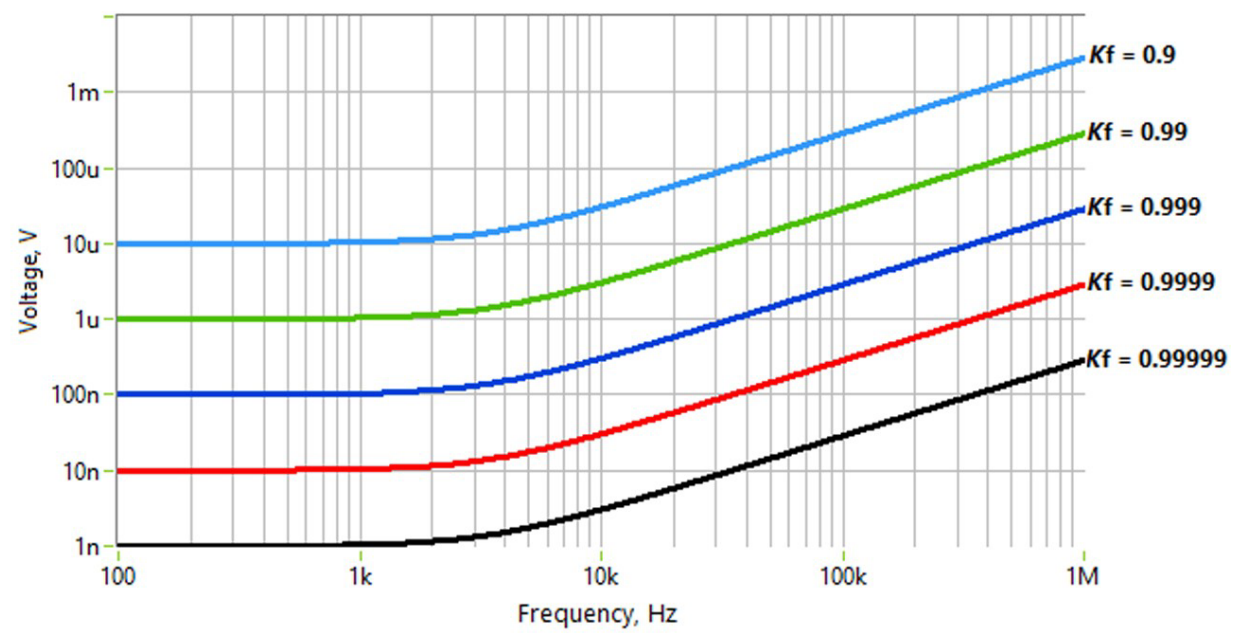

Fig. 2. Output voltage and frequency dependences of PGA207 instrumentation amplifier at different $K_{\mathrm{f}}$ values at common-mode $10 \mathrm{~V}$ and zero phase shift of the follower.

\section{Sample and hold circuit}

In order to achieve the required common-mode rejection, additional the sample and hold circuit can be used in the lock-in amplifier as shown in Figure 3.

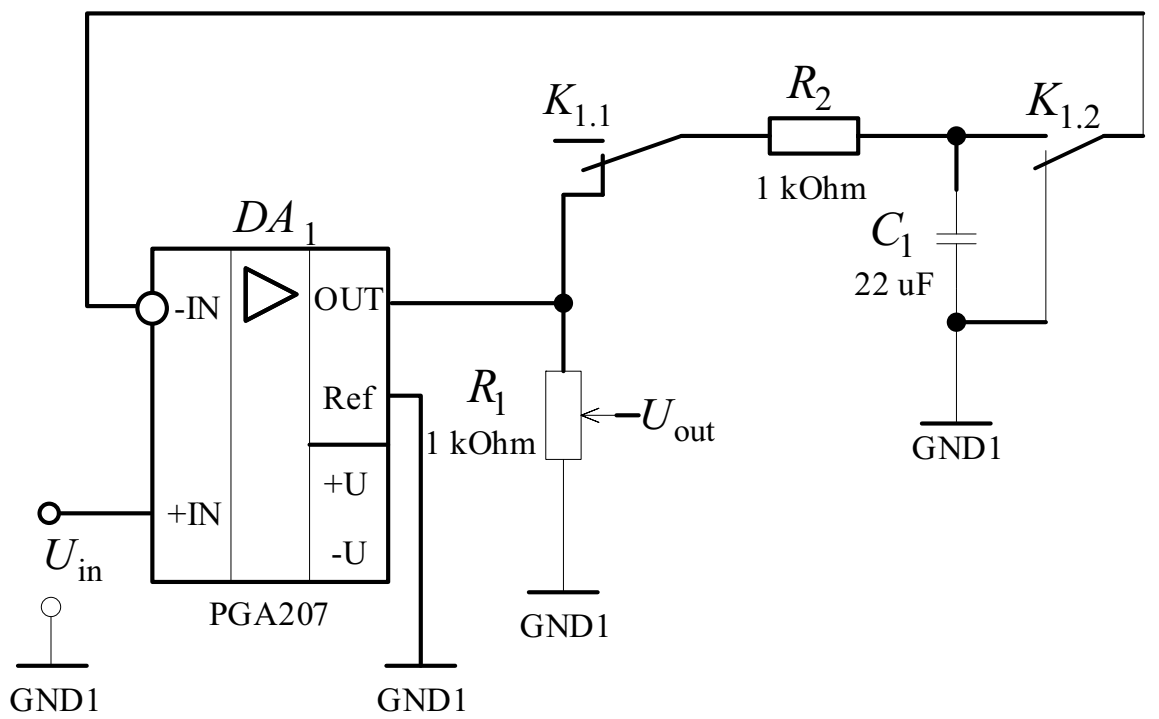

Fig. 3. Sample and hold circuit in PGA207. 
With this view, the switch $K_{1}$ (e.g. reed switch RES-55) is installed in the connection circuit of the instrumentation amplifier to supply measurement inputs of voltage $u_{0}(t)$. The discharge common-mode voltage processed by synchronous detector and low-pass filter is stored by condenser $C_{1}$ of the sample and hold circuit.

In the mode of measuring the differential signal, voltages $u_{0}(t)$ and $u_{x}(t)$ transmit to the measurements inputs of the instrumentation amplifier by switch $K_{1}$. At the same time, in the sample and hold circuit the voltage stored in the capacitor is subtracted from the input voltage.

\section{Conclusions}

The suggested procedures oriented towards the decrease of uncertainty of measuring the small differential signal against a large common-mode signal, meet the requirements of the up-to-date instrumentation.

The technical implementation suggested for the tracking symmetrical power supply for the instrumentation amplifier is based on the voltage follower that allows increasing the common-mode rejection ratio up to $160-180 \mathrm{~dB}$, comparing voltages up to $10 \sqrt{2} \mathrm{~V}$ within the $100 \mathrm{kHz}$ frequency range.

The work has been supported in part by the grant of the RFBR, research project No. 15-08-01007.

\section{References}

1. S. Avramov-Zamurovic, G.N. Stenbakken, A.D. Koffman, N.M. Oldham and R.W. Gammon, IEEE Trans. Instrum. Meas. 44, 904 (1995)

2. L. Callegaro, V. D'Elia, IEEE Trans. Instrum. Meas. 51, 632 (2002)

3. L. Callegaro D. Serazio, CPEM, 222 (2000)

4. J.C. Hsu, and Y. Ku, CPEM, 429 (2000)

5. A.V. Rogovyh, N.M. Natalinova, A.S. Spiridonova, A.S. Gordynets, SIBCON, 113590 (2015)

6. A. Dolgih, V. Martemyanov, SIBCON, 7491844 (2016)

7. V. Martemjanov, A. Dolgih (Ivanova), SIBCON, 6693632 (2013)

8. N. Natalinova, N. Ilina, E. Frantcuzskaia, IOP Conf. Series: Materials Science and Engineering, 132, 012029 (2016)

9. P.F. Baranov, E.I. Tsimbalist, V.N. Borikov, D.G. Soltanova, IMEKO, 116100 (2015)

10. P.F. Baranov, E.I. Tsimbalist, V.E. Baranova, SIBCON, 7146992 (2015)

11. M.L. Meade, Lock-in amplifiers: Principles and Applications (London: Peter Peregrinus Ltd, 1983)

12. S. Fu, A. Sakurai, L. Liu, F. Edman, T. Pullerits, V. Öwall, K. Karki, Rev. Sci. Instrum. 84, 115101 (2013)

13. M. Min, O. Martens, T. Parve, Measurement 27, 21 (2000) 